\title{
GENETICAL COMPOSITION, TEMPERATURE, DENSITY AND SELECTION IN AN ENZYME POLYMORPHISM
}

\author{
A. J. BIRLEY* and J. A. BEARDMORE $\uparrow$ \\ * Department of Genetics, University of Birmingham, P.O. Box 363, Birmingham B15 2TT \\ tDepartment of Genetics, University College of Swansea, Singleton Park, Swansea SA2 8PP
}

Received 1.xii.76

\section{Summary}

\begin{abstract}
Selection in egg-to-adult viability was investigated at the Esterase- 6 locus of Drosophila melanogaster. A factorial experiment was carried out with three variables, temperature, density and genetical composition; there were four temperatures $\left(15^{\circ}, 20^{\circ}, 25^{\circ}\right.$ and $\left.30^{\circ}\right)$, three densities $(115 \mathrm{ml}, 10 \mathrm{ml}$ and $2.5 \mathrm{ml}$ of food).

Strong frequency dependent selection was observed in many environments. The strength of the selection was very much modified by an interaction between temperature and density. The results are discussed in relation to evidence for a chemical mechanism which mediates frequency dependent selection, by conditioning, in the larval environment.
\end{abstract}

\section{Introduction}

STUdies of protein variation in a variety of species have shown that there is considerable genetical diversity both within and between experimental and natural populations of many species (Lewontin and Hubby, 1966; Harris, 1966, 1969; Selander and Yang, 1969). Such variation may be due to natural selection (Clarke, 1970; Richmond, 1970) or may be non-adaptive (King and Jukes, 1969; Kimura and Ohta, 1970).

In laboratory populations, some protein polymorphisms respond to natural selection (Powell, 1971; McDonald and Ayala, 1974; Minawa and Birley, 1975), all working with species of Drosophila. Indeed, these results show that natural selection determines a substantial fraction of the observed genetical diversity within laboratory populations. Studies of the Adh locus in Drosophila melanogaster by Clark (1975) and his colleagues have shown ways in which selection could operate directly on allozymes and selection in enzyme polymorphisms has also been observed in nature. For example, the temperature associated clinal variation in allele frequency at a lactate dehydrogenase locus in the crested blenny, Anoplarchus purpurescens (Johnson, 1971) and at an Esterase locus in Catastomus clarkii (Koehn, 1969), support the selectionist viewpoint. An important feature of the latter study is that the in vitro activities of the two allozymes are correlated with temperature, the putative selective factor. On the other hand, some authors (Kimura and Ohta, 1970) assume that selective coefficients are in general small. If this were so selection would be difficult to detect by experiment. Indeed, the results of at least one closely controlled laboratory experiment has, despite a detailed analysis, shown only weak, if any, selection (Yamazaki, 1971). If the mechanism of evolution is to be understood it is important that the magnitude, nature and extent of the selective forces which operate within populations are assessed upon a range of protein polymorphisms.

It has been argued that a positive relationship is likely to exist between the extent of the genetical diversity within a population and the heterogeneity 
of the environment it inhabits (Ludwig, 1950; Dobzhansky, 1951; Mayr, 1963). In the short term, variable environments have been shown to favour a higher level of genetical variability of a polygenic kind (Beardmore and Levine, 1963; Long, 1970) and of heterozygosity at some protein loci (Powell, 1971; McDonald and Ayala, 1974). Moreover, theoretical studies have shown that variation in the environment can, under certain conditions, maintain genetical variability within populations (MaynardSmith, 1966; Hoekstra, 1975).

Frcquency dependent selection may be mediated through environmental variability and/or competition (Mather, 1969). Tobari and Kojima (1969) demonstrated frequency dependent selection in association with the Adh locus of Drosophila melanogaster as did Kojima and Yarbrough (1967) with the Esterase-6 locus. We have previously shown that allele frequencies at the Esterase-6 locus are associated with temperature and density in laboratory populations of common origin. The correlation with temperature was seen in populations from either constant or fluctuating temperature environments (Beardmore, 1970; Birley, unpublished). The population, used by Kojima and Yarbrough (1967) for a study of selection at the Esterase-6 locus, was established from a cross between two inbred lines. There is then the possibility that the selection which they observed was modified by a lack of genetic co-adaptation or a failure of the synthetic population to reach linkage equilibrium. The population used in this study originated from nature and was used to investigate the mode of selection, in relation to temperature and density, at the Esterase-6 locus of Drosophila melanogaster.

\section{Materials and methods}

The population used in this study was established and maintained in a Perspex population cage (Beardmore, Van Delden and Alkema, 1963), by Dr T. C. Long in May 1966. It was derived from the progeny of nine inseminated female flies captured in Groningen, the Netherlands. Food medium was supplied in $35 \mathrm{ml}$ lots in polyethylene cups and was prepared according to the recipe given by Mittler and Bennett (1962). The population received two new cups every 3 days. Polyacrylamide gel electrophoresis of individual flies from the population showed it to be segregating for two alleles at the Esterase-6 locus, namely $E s t-6^{F}$ and $E s t-6^{S}$. We have abbreviated the nomenclature of the three genotypes in the Esterase-6 polymorphism as follows: $E s t-6^{F^{\prime} F}=F F, E s t-6^{F^{\prime} S}=F S$ and $E s t-6 S^{\prime} S=S S$. Allele frequencies are given in terms of the $E s t-6^{F}$ allele, e.g. $0 \cdot 8(F)$.

Six lines homozygous for the $E s t-6^{F}$ allele and 10 lines homozygous for the $E s t-6 s$ allele were extracted from the population. Cultures differing in their genetical composition were established in the way described by Kojima and Yarbrough (1967). Three genetical compositions were used, $0 \cdot 8(F), 0 \cdot 5(F)$ and $0 \cdot 2(F)$. Two hundred fertilised females were mixed together such that the expected zygotic proportions of the Esterase-6 genotypes would be in Hardy-Weinberg proportions. The females were allowed to lay eggs for 50 hours at $25^{\circ} \mathrm{C}$. Cultures were then raised in all combinations of four temperatures, and three densities. The three densities were defined in terms of food volume per culture vessel (low $115 \mathrm{ml}$; medium $10 \mathrm{ml}$; high $2.5 \mathrm{ml}$ ). The four temperatures used were $15^{\circ}, 20^{\circ}, 25^{\circ}$ and $30^{\circ}$. Four replicate cultures were raised in each of the 36 combinations of density and 
temperature. About 48 adults were sampled at random from all of the emergents from individual cultures, and typed for their Esterase- 6 genotype.

Polyacrylamide gel electrophoresis was carried out using the disc

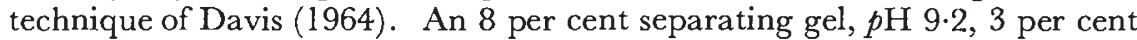
spacer gel $p \mathrm{H} 6.7 \quad(0.5 \mathrm{~cm}$ long) and 3 per cent sample gel were used. Electrophoresis was carried out at $4^{\circ} \mathrm{C}$ with a constant current of $2 \mathrm{~mA}$ per gel column, until the bromophenol blue marker had migrated to a point $0.5 \mathrm{~cm}$ from the base of the gel columns. After electrophoresis, the gels were equilibriated in $0.1 \mathrm{M}$ phosphate buffer $p \mathrm{H} 6.0$ at $4^{\circ} \mathrm{C}$ for 30 minutes. The Esterase phenotypes were revealed with $\alpha$-naphthyl acetate $(1 \mathrm{mg} / \mathrm{ml})$ as a substrate and Fast Blue RR $(0.1 \mathrm{mg} / \mathrm{ml})$ in $0.1 \mathrm{M}$ phosphate buffer $p \mathrm{H} 6.0$ for 30 minutes at $25^{\circ} \mathrm{C}$.

\section{Results}

A chi-square test was used to compare the genotype frequencies from replicate cultures. Except for those cultures raised at $15^{\circ}$, low density and $0.8(F),(P=0.02)$, replicate cultures had similar genotypic frequencies; with an experiment of this size a single case of heterogeneity could arise by chance alone. Subsequent statistical analyses were carried out with the pooled genotypic frequencies from replicate cultures. Test statistics were provided by the log likelihood ratio, (2I or G-test (Kullback, 1959; Sokal and Rohlf, 1969)). Table 1 shows the output genotype frequencies for all combinations of temperature density and genetical composition. The $G$-values also shown in table 1 , were calculated for a comparison of the observed and expected genotype frequencies in all 36 conditions. The expected frequencies contain the assumption that the eggs laid by the fertilised female parents were in Hardy-Weinberg proportions. Although the fecundity of females in this population had been shown to vary with age and genotype (Birley and Beardmore, 1972), in this case a control experiment measuring the fecundity of the 3-4-day-old female parents failed to detect any difference between Esterase- 6 homozygotes. The values of $G$ when compared with the $\chi^{2}$-distribution for 2 d.f. show a wealth of evidence in favour of natural selection in egg-to-adult viability. The total variation in observed and expected genotype frequencies is measured by the sum of the 36 individual $G$ values for 2 d.f. given for 72 d.f. a $G$-value of 1864.492. Further analysis of the data was carried out by partitioning the 72 d.f. into items measuring the homogeneity of genotype frequencies $(l)$, in relation to the three variables, temperature $(i)$ density $(j)$ and genetical composition $(k)$. The analysis is outlined below and follows the methods given by Kullback (1959).

Let the observed genotype frequency in any condition be represented by $n_{i j k l}$, where $i=1, q ; j=1, r ; k=1, s ; l=1, t$. If $\mathcal{N}$ is the total number of sampled individuals then

$$
N=\sum_{i} \sum_{j} \sum_{k} \sum_{i} n_{i j k l}
$$

The number of individuals sampled in any one condition (and fixed by the experimental design) may be represented as

$$
n_{i j k}=\sum_{i} n_{i j k} \text {. }
$$

The theoretical probabilities vary with genetical composition, and are represented as $p_{k l}$ where as above $k=1, s$ and $l=1, l$. The form and 

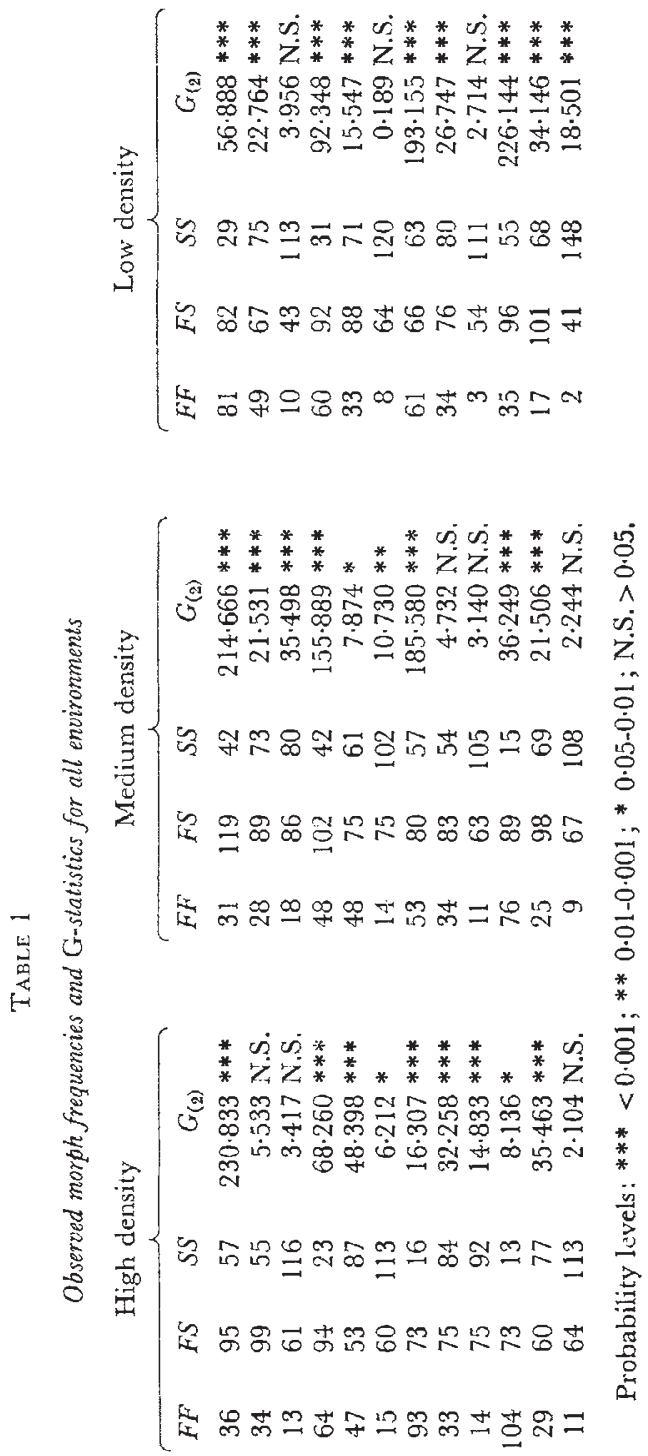

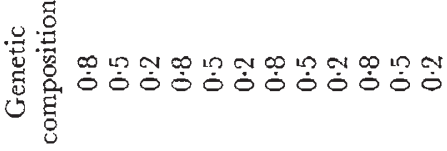

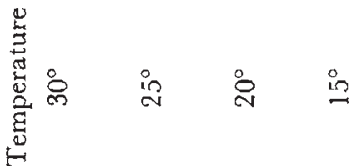


results of the analysis are shown in table 2. The degrees of freedom follow those of the $\chi^{2}$-distribution test and algebraic derivations of the test statistic $(G$-value) are seen under the heading "Information". Numerical evaluation of the $G$-value was carried out after formulae had been re-arranged into a form more suitable for computation. For example, the effect of density is given as

$$
G=2 \sum_{j} \sum_{l} n . j . i \log \frac{n \cdot j . l N}{n \cdot j . . n \ldots l} .
$$

On rearrangement, this expression becomes:

$$
G=2 \sum_{j} \sum_{l} n . j . l \log n . j . l-2 \sum_{j} n . j . . \log n . j . .-2 \sum_{i} n . . . l \log n . . . l+N \log N .
$$

The results of the statistical analysis (table 2) show a highly significant difference between the observed and expected genotype frequencies. This item represents the extent of the overall deviation of observed and expected genotype frequencies irrespective of the effects of temperature and density; each of the three genetical compositions provides 2 of the total 6 degrees of freedom. Regardless of genetical composition and density, it is seen that the genotype frequencies from the four temperatures can be regarded as homogeneous. But, the item Temperature $\times$ Genetical composition, shows that the homogeneity between the temperatures varies with the genetical composition of cultures. The statistical significance of this item is however, slight compared with the high level of statistical significance shown by the remaining four partitions. There is a highly significant effect of density alone, and the effect of density varies with genetical composition. There are also high orders of heterogeneity in the genotype frequencies. The main effects, temperature and density, do not have an independent effect upon genotype frequency, and this association varies with the genetical composition. This means that genotypic fitness is the result of interaction between the two variables temperature and density. The heterogeneity between the effects of temperature and density and genetical composition does not define the nature of the relationships between genotypic fitness and genetical composition. This is because the response to selection, as measured by the $G$-values will vary with genetical composition, irrespective of whether fitnesses are constant (e.g. heterozygote advantage) or variable (e.g. frequency dependent selection). Insight into the nature of this interaction and of the mode of selection may be gleaned from figs. I and 2. Here a simple measure of fitness is used to compare the viabilities of the Esterase-6 genotypes. It is essentially the same as that used by Kojima and Yarbrough (1967), and is the ratio of output $(O)$ to input genotype proportions $(I)$ or $O / I$ value. The $O / I$ values are plotted for each genotype, in the relation to the three genetical compositions, for all 12 combinations of density and temperature in figs. 1 and 2. An outline of the general features of these results is given in this report. A pronounced inverse relationship between the input frequency of morphs and their fitness is a general (i.e. not invariable) feature of the results. The $O / I$ value of the $F F$ homozygote is highest in $0 \cdot 2(F)$ cultures and lowest in $0 \cdot 8(F)$ cultures. The $O / I$ value of the $S S$ homozygote is greatest in $0 \cdot 8(F)$ and lowest in $0 \cdot 2(F)$ cultures. Also, the $O / I$ value of the heterozygote is often lowest and less than unity in $0 \cdot 5(F)$ cultures, where its input frequency is maximal. Hence, the general mode of selection is frequency dependent but the strength of the selection is modified by an interaction 

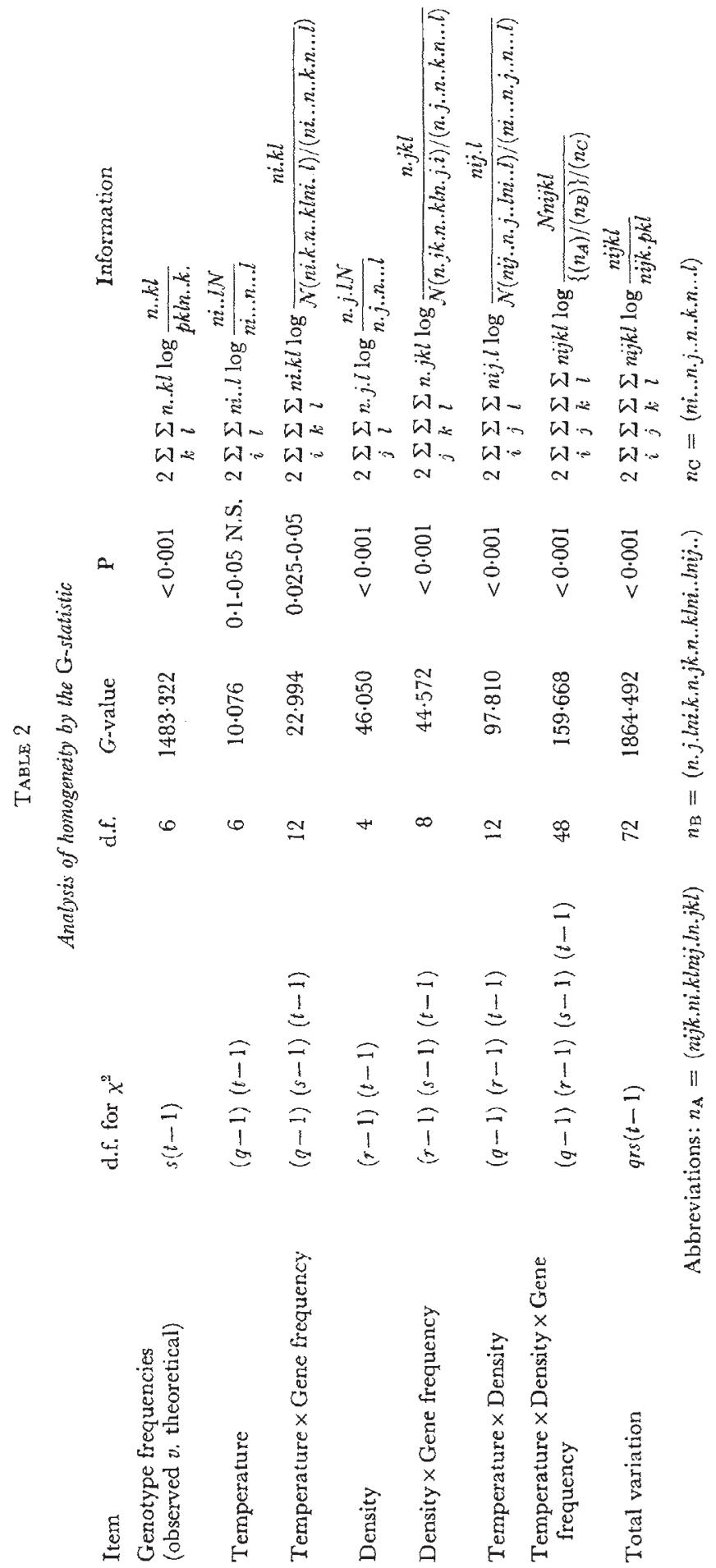
between temperature and density. For example, the strength of the frequency dependent selection particularly in $0 \cdot 8(F)$ cultures, decreases at $30^{\circ}$ with an increase of density. At $15^{\circ}$ the reverse is seen; selection is greatest at high density. Generally, the effect of medium density is one of transition between the high and low density conditions. The effect of temperature and genetical composition is also transitional between $30^{\circ}$ and $15^{\circ}$, at $25^{\circ}$ and $20^{\circ}$. Selection is strongest in $0 \cdot 8(F)$ cultures, a genetical composition which is furthest away from the " equilibrium " gene frequency of the mother cage population. In $0 \cdot 2(F)$ cultures selection is much less strong than in $0.8(F)$ cultures, and in some conditions, notably at low density, when the temperature is $15^{\circ}$ or $20^{\circ}$ the $O / I$ value of the $F F$ homozygote is never greater than that of the SS homozygote. In summary, the general features of these results are as follows:

1. Selection in egg-to-adult viability at the Esterase-6 locus can be very strong.

2. Frequency dependent selection of a type that would lead to a " stable" equilibrium is seen in many but not all conditions.

3. The effects of temperature and density are not independent upon genotypic fitness, and vary with genetical composition.
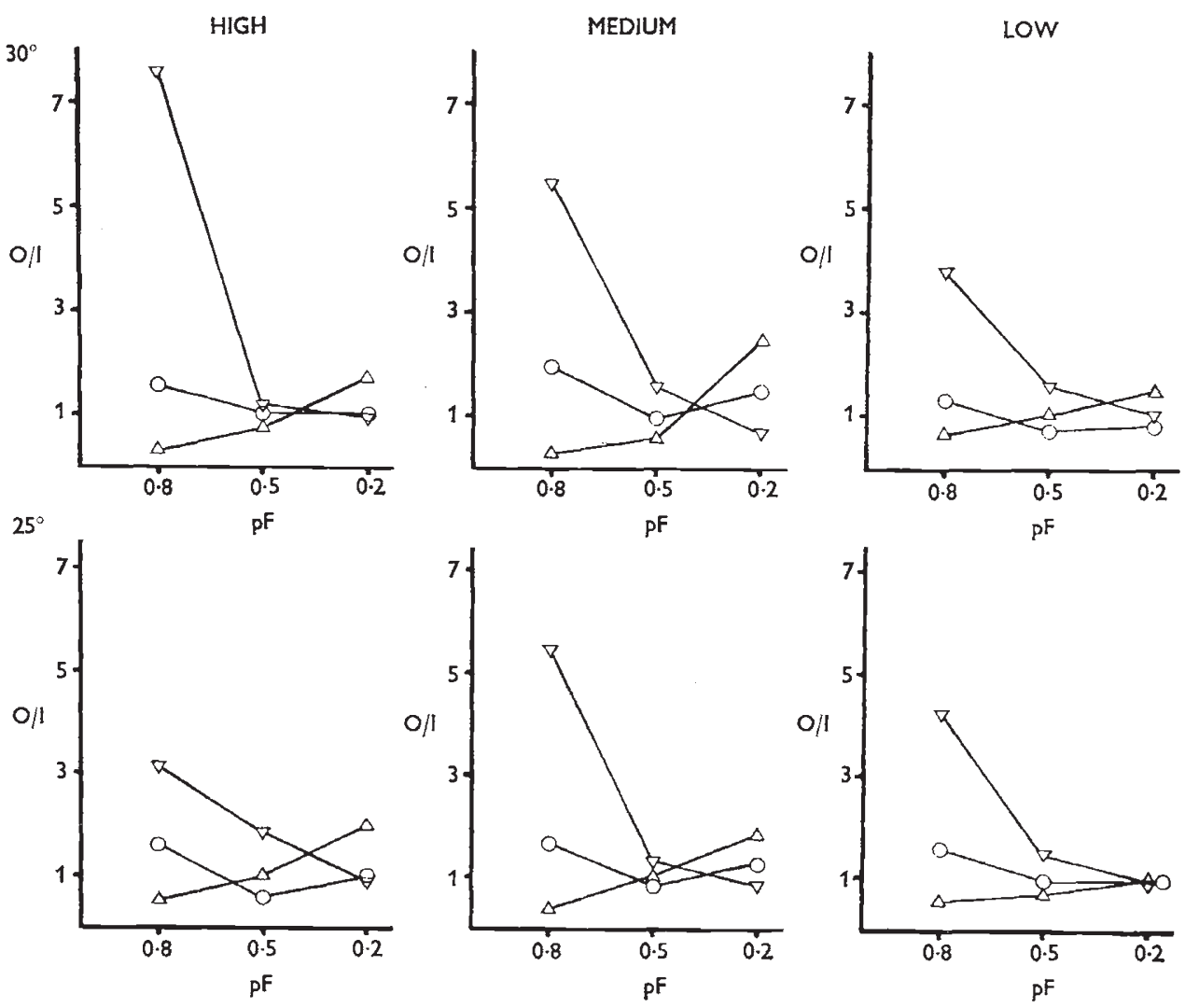

FIG. 1.-O/I values plotted in relation to genetical composition $(p F)$ and density, at $30^{\circ}$ and

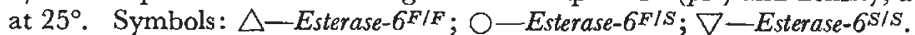




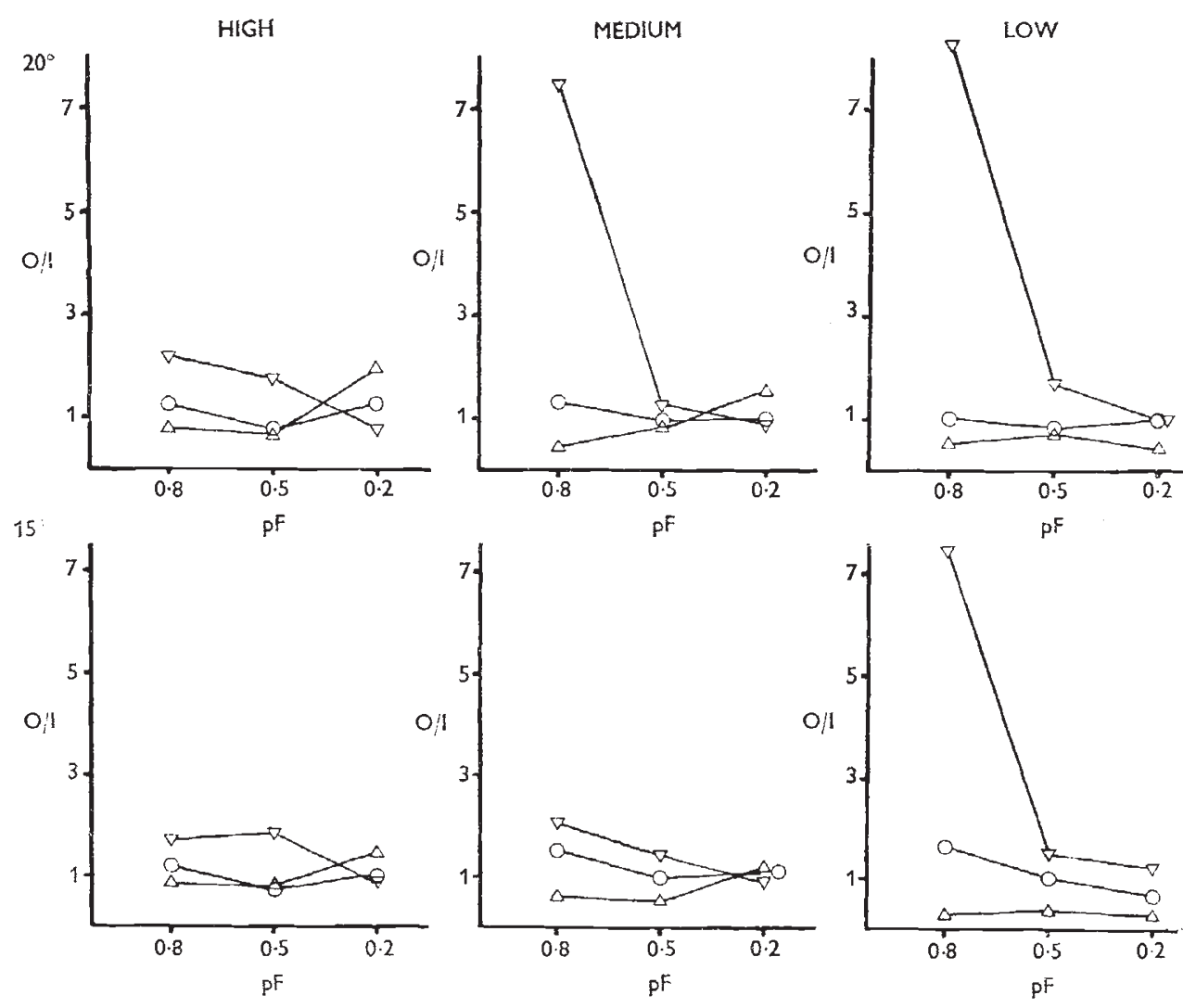

Fis. 2.--O/I values plotted in relation to genetical composition $(P F)$ and density at $20^{\circ}$ and

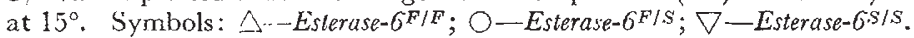

\section{Discussion}

The population used in this study had been maintained in the cage environment at $25^{\circ}$ since May 1966, and was stable with respect of the Esterase-6 polymorphism. Adults were sampled from the population cage in November 1968 and in August 1969 and wcre typed for thcir Esterase-6 genotypes. The rcsults are given in table 3 . A contingency $\chi^{2}$ test for 2 d.f. with this data gave a value of $1.07(\mathrm{P}>0.5)$. The samples may be regarded as homogeneous and a common estimate of $E^{\prime} t-6^{F}$ allele frequency is $0 \cdot 156$. The cxtraction of lines homozygous at the Esterase-6 locus was made over this period and the experiment carricd out in summer 1969. Populations

TABIE 3

Estcrase-6 genotype frequencies in the experimental cage populations

\begin{tabular}{|c|c|c|c|c|}
\hline \multirow[b]{2}{*}{ Sampling timc } & \multicolumn{3}{|c|}{ Genotype } & \\
\hline & $E s t-6 k^{\prime \prime} k^{\prime}$ & $E s t-6 \sigma^{F^{\prime}} S$ & Est-6,s's & Total no. \\
\hline & 8 & 53 & 163 & 224 \\
\hline Augus & 2 & 27 & 67 & 96 \\
\hline
\end{tabular}


with the same ancestry and establishment time, as that used for this study have shown the following features (Beardmore, 1970; Birley, unpublished).

(a) The equilibrium frequency of the Esterase- $6^{F}$ allele is negatively correlated with temperature in thermally stable populations $\left(17.5^{\circ}, 20^{\circ}, 25^{\circ}\right.$ and $29^{\circ}$.

(b) Regular oscillations in allele frequency occur in populations kept in fluctuating temperature environments (sinusoidal periods of 28 and 96 days, maximum temperature $30^{\circ}$, minimum $20^{\circ}$ ). The pattern of change in allele frequencies in such populations can be predicted from the constant temperature populations. Selection involving the Esterase-6 polymorphism of Drosophila melanogaster has been demonstrated by a number of workers (MacIntyre and Wright, 1966; Kojima and Yarbrough, 1967; Huang et al., 1971). The major component of fitness in this and the latter two studies was egg-to-adult viability. This study like that of Kojima and Yarbrough (1967) has demonstrated frequency dependent selection at least in association with genetical polymorphism at the Esterase-6 locus of Drosophila melanogaster. The detailed discussion of the results is divided into two sections: (a) the basic frequency dependent response to selection, and (b) modification related to an interaction of density and temperature.

Insight into a possible ecological mechanism for frequency dependent selection was obtained by Huang et al. (1971). In their experiment the fitness of Esterase-6 genotypes was dependent upon the genotypic nature of the previous habitation of the larval environment. Their conclusions were obtained from a comparison of productivities of Esterase- 6 genotypes raised upon food media which had or had not been conditioned by larvae of the same or different Esterase-6 genotype. We have carried out similar experiments to those of Huang et al., and our results are in agreement; larval conditioning can mediate frequency dependent selection in egg-to-adult viability. In addition, there is chemical evidence which supports the mechanism of larval conditioning. Lines homozygous for an Esterase-6 allele produce a distinct chemical in large quantities into the food medium. The substances are isoamyl alcohol and p-hydroxybenzoic acid for Est-6S/S and $E s t-6^{F} / F$ homozygotes respectively. Each substance is only inhibitory in fitness, to the producer homozygote (Naylor, Beardmore and Ballantyne, unpublished). Heterozygotes, Est-6F/S, produce both chemicals in smaller quantity. The production of these chemicals is consistent for a wide range of Drosophila melanogaster strains, and also for the sibling species Drosophila simulans (Naylor and Beardmore, unpublished). The Esterase-6 polymorphism is ubiquitous in both $D$. melanogaster and $D$. simulans. The common allele in D. melanogaster is electrophoretically slow and that in $D$. simulans the electrophoretically faster migrating form. It is of interest to note that the same chemical specifity for the fast and slow alleles applies to $D$. simulans. There is then a decided consistency in the mechanism of frequency dependent selection for between sibling species by larval conditioning at this locus.

Consider the interaction of density and temperature upon the pattern of frequency dependent selection. Temperature has already been implicated in the maintenance of polymorphism, at the Esterase- 6 locus in laboratory cage populations. But, the "equilibrium" allele frequencies as predicted by this experiment do not correspond with those of the cage populations. This conclusion is based upon results at medium density (figs. 1 and 2) since this density closely corresponds, in terms of individual survival, to the cage 
condition; it is seen that $E s t-6^{F}$ allele frequency would increase rather than decrease with temperature rise. The outcome may be different for total fitness; nonetheless, temperature is not the causal factor for the results in thermally different environments. Selection by density dependence and frequency dependence can be interrelated (Turner and Williamson, 1968). Larval density and competition for food are also associated and can be expressed in development time (Bakker, 1965, 1969; Kearsey, 1961). The interaction between density and temperature with genetical composition is seen in pure cultures of the Esterase- 6 genotypes, both in culture productivity and development time (Birley and Beardmore, 1972). Differences in development time are also manifest in the populations; fig. 3 shows a marked

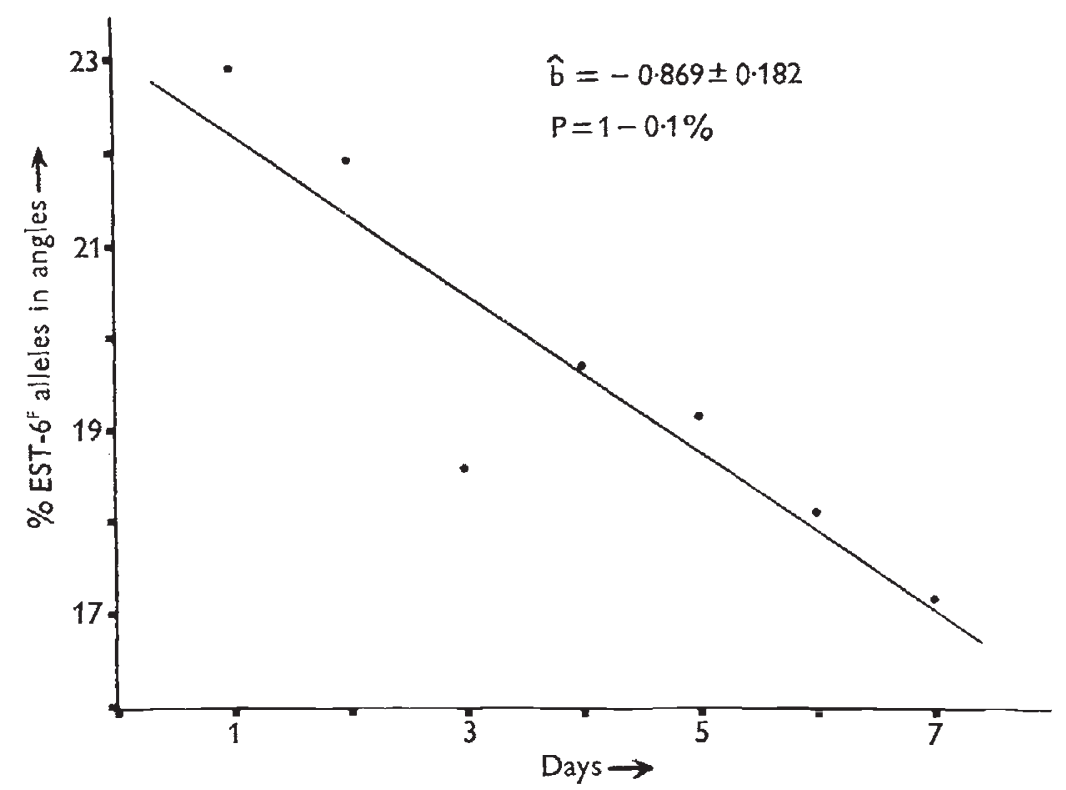

Frg. 3.--Regression of emergence time upon percentage Esterase- $\sigma^{F}$ alleles, in a polymorphic culture. Day 1 is the first day of emergence.

relationship between the $E s t-6^{F}$ allele frequency and dcvelopment time amongst adults from a population food cup. The implications are that at least three non-independent factors are involved in the mechanism of selection, larval conditioning, temperature and density. This raises the problem of whether such apparcnt complexity is due to pleiotropy of the Esterase-6 locus per se or is due to linkage disequilibrium between alleles at the Esterase-6 locus and gencs which contribute to these continuously variable characters, or components of Darwinian fitness. Linkage disequilibrium can arise through sampling variation, for example in the sample of parents used to initiate a cage population, and in the sample of lines homozygous for the $E s t-6^{F}$ or $E s t-6^{S}$ alleles which were used as a basis for the experiment. Obviously the smaller the sample the greater the recombination fraction over which a significant disequilibrium could arise because of such variation. Alternatively, linkage disequilibrium may arise through selection in the cage population, it may indeed persist in long-established and large laboratory 
populations (Birley, 1974). There is a gross dissimilarity between the genetical background of the Dutch population used in this study, and that of the population used by Kojima and Yarbrough (1967), yet there are strong similarities in the observed mode of selection. Although Dolan and Robertson (1975) were unable to demonstrate larval conditioning amongst strains with extremely diverse genetical backgrounds, larval conditioning does occur when the genotypes tested are all derived from the same outbred or evolving laboratory populations. It is concluded that the ubiquitous Esterase-6 polymorphism is a component of the genetical architecture which confers adaptation to the environment by frequency dependent selection. The interrelationships between ecological factors which mediate frequency dependent selection suggests that the effects at the Esterase-6 locus are evolved with linkage disequilibrium. The apparent generality of the mechanism in outbred populations suggests that further physiological and genetical studies of frequency dependent selection are worthy of further study.

Acknowledgments.-Dr Paul Davies of the Department of Mathematical Statistics, University of Birmingham, is thanked for his advice concerning the log likelihood ratio test statistic.

\section{REFERENCES}

BAKKER, K. 1961. An analysis of factors which determine success in competition for food among larvae of Drosophila melanogaster. Arch. Neerl. de. Zool., 14, 200-281.

BAKKER, K. 1969. Selection for rate of growth and its influence on competitive ability on the larvae of Drosophila melanogaster. Arch. Neerl. de Zool., 19, 541-595

BEARDMOrE, J. A., AND Levine, L. 1963. Fitness and environmental variation. I. A study of some polymorphic populations of Drosophila pseudo-obscura. Evolution, 17, 121-129.

BEARDMORE, J. A. 1970. Ecological factors and the variability of gene-pools in Drosophila. In Essavs in Evolution and Genetics in Honour of Theodosius Dobzhansky, ed. M. K. Hecht and W. C. Steere, pp. 299-314.

BEARDMORE, J. A., W. VAN DELDEN, AND L. ALKEMA. 1963. A population cage for incubator use. Drosophila Information Service, 37, 137-138.

BIRLEY, A. J. 1974. Multi-locus polymorphism and selection in a population of Drosophila melanogaster. I. Linkage disequilibrium on chromosome III. Heredity, 32, 122-127.

BIRLEX, A. J., AND BEARDMORE, J. A. 1972. Manifold large selective effects in an enzyme polymorphism. 5th European Mar. Biol. Symp. Piccin Editore, Padua, 81-100.

GLARKE, B. C. 1970. Selective constraints on amino-acid substitutions during the evolution of proteins. Nature. 228, I59-160.

CLARKE, B. C. 1975. The contribution of ecological genetics to evolutionary theory: detecting the direct effects of natural selection on particular polymorphic loci. Genetics, 79, 101-113.

DAvys, B. J. 1964. Disc electrophoresis. II. Method and application to human serum proteins. Ann. N.Y. Acad. Sci., 121, 404-427.

DOLAN, R., AND ROBERTSON, A. 1975. The effect of conditioning the food medium in Drosophilia in relation to frequency dependent selection. Heredity, 35, 31 I-316.

Dobzhansky, TH. 1951. Genetics and the Origin of Species, 3rd Edition. Columbia University Press.

HARRIS, H. 1966. Enzyme polymorphisms in man. Proc. Roy. Soc. B, 164, 298-310.

HARris, H. 1969. Genes and isozymes. Proc. Roy. Soc. B, 174, 1-31.

HUANG, s. L., SINGH, M., AND KOJMA, K. 1971. A study of frequency-dependent selection observed at the Esterase- 6 locus of Drosophila melanogaster using a conditioned media method. Genetics, 68, 97-104.

hoekstra, R. 1975. A deterministic model of cyclical selection. Genet. Res. Camb., 25, 1-15. JoHnson, M. s. 197I. Adaptive lactate dehydrogenase variation in the crested blenny, Anoplarchus purpurescens. Heredity, 27, 205-226.

KEARSEY, M. J. 1965. The interaction of competition and food supply in the two lines of Drosophila melanogaster. Heredity, 20, 169-181. 
KIMURA, M., AND OITA, T. 1970. Protein polymorphism as a phase of molecular evolution. Nature, 229, 467-469.

KING, J. L., AND JUKES, T. H. 1969. Non-Darwinian evolution. Science, 164, 788-798.

KOEHN, R. K. 1969. Esterase heterogeneity: dynamics of a polymorphism. Science, 163, 943-944.

Kojima, K., AND yarbrougi1, к. M. 1967. Frequency dependent selection at the Esterase-6 locus in Drosophila melanogaster. Proc. Nat. Acad. Sci., 57, 645-649.

kULlback, s. 1959. Information Theory and Statistics. Jolin Wiley and Sons Inc., New York.

LEWONTIN, R. C., AND HUBBY, J. x. 1966. A molccular approach to the study of genic heterozygosity in natural populations. II. Amount of variation and degree of heterozygosity in natural populations of Drosophila pseudo-obscura. Genetics, 54, 595-605.

LONG, T. 1970. Genetic effects of fluctuating temperature in populations of Drosophila melanogaster. Genetics, 60 401-416.

Ludwig, w. 1950. Zur theorie der Konkurrenz. Dic Annidation (Einnischung) als fünftcr Evolutions faktor. Neue Ergeb. Probleme Zool., Klatt-Festschrift, 516-.537.

MAGINTYRE, R. J., AND WRIGHT, T. R. F. 1966. Responses of Esterase-6 alleles of Drosophila melanogaster and Drosophila simulans to selection in experimental populations. Genetics, $53,371-375$.

MCDONAI.D, J. F., AND AYAT,A, F. J. 1974. Genctic response to environmental hetcrogeneity. Nature, 250, 572-574.

MATHER, K. 1969. Selection through competition. Heredity, 24, 529-540.

maynard-smirh, J. 1966. Sympatric speciation. Amer. Nat., 100, 637-650.

MAYR, 1.. 1963. Animal Species and Evolution. Oxford Univcrsity Press.

MINAWA, A., AND BIRLEY, A. J. 1975. Genetical and environmental diversity in Drosophila melanogaster. Nature, 255, 702-704.

MITTLER, S., AND BENNETT, J. 1962. A simple food medium that requircs no live yeast with the minimum of variables. Drosophila Information Service, 36, 131-132.

pOWELL, R. J. 1971. Genetic polymorphisms in varied environments. Science, 174, 10351036.

RICHMOND, R. C. 1970. Non-Darwinian evolution: a critique. Nature, 225, 1025-1028.

SELANDER, R. K. AND YANG, S. Y. 1969. Protein polymorphism and genic heterozygosity in a wild population of the house mouse (Mus musculus). Genetics, 63, 653-667.

SOKAL, R. R., AND ROHLF, F. J. 1969. Biometry: The Principles and Practice of Statistics in Biological Research. Chapter 16, Analysis of frequencies. W. H. Frecman and Company, San Francisco.

TOBARI, Y. N., AND KOJIMA, K. 1969. The pattern of viability changes associated with genotype frequency at the alcohol dehydrogenase locus in a population of Drosophila melanogaster. Genetics, 61, 201-209.

TURner, J. R. G., AND Williamson, M. H. 1968. Population size, natural selection and genetic load. Nature, 218, 700.

yama7aki, T. 1971. Measurement of fitness at the Esterasc-5 locus in Drosophila pseudoobscura. Genetics, 67, 579-603. 